\title{
On the asymptotic behavior of Einstein manifolds with an integral bound on the Weyl curvature
}

Romain Gicquaud, Dandan Ji and Yuguang Shi

In this article, we consider the geometric behavior near infinity of some Einstein manifolds $\left(X^{n}, g\right)$ with Weyl curvature belonging to a certain $L^{p}$ space. Namely, we show that if $\left(X^{n}, g\right), n \geq 7$, admits an essential set, satisfies Ric $=-(n-1) g$, and has its Weyl curvature in $L^{p}$ for some $1<p<\frac{n-1}{2}$, then the norm of the Weyl tensor decays exponentially fast at infinity. One interesting application of this theorem is to show a rigidity result for the hyperbolic space under an integral condition for the curvature.

1 Introduction

2 Basic estimates

3 Pointwise estimate for the Weyl tensor

4 Applications

Acknowledgments

References

\section{Introduction}

During the last three decades there were lots of interesting works on the asymptotic behavior of Ricci flat metrics with integral bounds on curvature. See, e.g., [5, 10]. These works gave an nice intrinsic characterization of asymptotic locally Euclidean (ALE) manifolds. Inspired by these works, we want to study a similar problem in the context of asymptotic locally hyperbolic (ALH) manifolds. The ALH case appears much more complicated than 
the ALE case in both geometric and analytic parts. As an example, the rescaling argument which is very efficient in the analysis of the asymptotic behavior of ALE metrics does not work in the ALH case because the model is the hyperbolic metric which is not scale invariant. Another complication arises from the Möbius group of $\mathbb{S}^{n}$ which allows cuspidal ends. To rule out such ends, we need to assume that the manifold $\left(X^{n}, g\right)$ admits an essential set (see [4] for more details):

Definition 1.1. A non-empty compact subset $\mathbb{D}$ of a complete non-compact Riemannian manifold $\left(X^{n}, g\right)$ is called an essential set if

1 . $\mathbb{D}$ is a compact domain of $X^{n}$ with smooth and strictly convex boundary $\mathbf{B}:=\partial \mathbb{D}$, i.e., its second fundamental form with respect to the outward unit normal vector field is positive definite;

2. $\mathbb{D}$ is totally convex: there is no geodesic $\gamma:[a, b] \rightarrow X$ such that $\gamma(a)$, $\gamma(b) \in \mathbb{D}$ and $\gamma(c) \notin \mathbb{D}$ for some $c \in[a, b] ;$

3. the sectional curvature of $\left(X^{n}, g\right)$ is negative outside $\mathbb{D}$.

Assuming that $\left(X^{n}, g\right)$ is hyperbolic the existence of an essential set is equivalent to the requirement that $\left(X^{n}, g\right)$ is convex and co-compact. More generally, it can be shown that conformally compact and Cartan-Hadamard manifolds admit essential sets; see [14]. Together with assumptions on the rate of convergence of the sectional curvature to -1 at infinity, the existence of an essential set has been used to prove the existence and the regularity of a conformal compactification of the manifold $\left(X^{n}, g\right)$ in $[2,3,16,20]$.

In what follows, we define $\rho: X \rightarrow \mathbb{R}$ as the distance function from $\mathbb{D}$ :

$$
\rho:=d_{g}(\mathbb{D}, \cdot)
$$

In [4], it has been proven that, if $\mathbb{D} \subset X$ is an essential set, $\rho$ is a smooth function on $X^{n} \backslash \mathbb{D}$ and has no critical point. This implies that $X \backslash \mathbb{D}$ is diffeomorphic to $[0, \infty) \times \mathbf{B}$.

In this paper, we want to investigate the behavior at infinity of some complete non-compact Einstein manifolds with

$$
\operatorname{Ric}_{g}=-(n-1) g \text {, }
$$

with Weyl curvature belonging to a certain $L^{p}$-space and contanining an essential set $\mathbb{D}$. In particular, we show that they are asymptotically locally hyperbolic Einstein (ALHE) metric outside $\mathbb{D}$, meaning that $\sec _{g}+1=$ 
$O\left(\mathrm{e}^{-a \rho}\right)$ for some $a>0$. In contrast with the ALE case, the major difficulty in the ALH setting is the lack of sharp global Sobolev inequalities which are crucial in applying Moser iterations in the ALE case (see, e.g., $[5,10]$ ).

However, we observed a nice $L^{2}$-estimate for the Laplace operator acting on 4-tensors satisfying properties analogous to those of the Weyl tensor for manifolds of dimension greater than 5 , see Lemma 2.8 below. Thanks to this lemma and combining other techniques, we were able to obtain the following result (see also Theorem 3.4):

Theorem 1.2. Let $\left(X^{n}, g\right), n \geq 7$, be a complete non-compact Einstein manifold with

$$
\text { Ric }=-(n-1) g .
$$

Assume that $X^{n}$ contains an essential set $\mathbb{D}$. We denote $W$ the Weyl tensor of the metric $g$. If $\|W\|_{L^{p}\left(X^{n}, g\right)}<\infty$ for some $p \in\left(1, \frac{n-1}{2}\right)$, then there exists a constant $C$ such that

$$
|\mathrm{Rm}-\mathbf{K}| \leq C \mathrm{e}^{-(n+1) \rho} .
$$

Here $\mathrm{Rm}$ is the curvature tensor of the metric $g$ and $\mathbf{K}$ the constant curvature tensor with sectional curvature -1 with respect to metric g, i.e.,

$$
\mathbf{K}_{i j k l}=-\left(g_{i k} g_{j l}-g_{i l} g_{j k}\right) .
$$

Remark 1.3. In order to get the curvature estimate (1.1) we need a variant of the maximum principle, this is the reason why we assumed $n \geq 7$, we believe that the theorem should be true for $n=5,6$ and will address this in a future paper.

Since $\left(X^{n}, g\right)$ is Einstein and has positive injectivity radius, it will become apparent that $\mathrm{W} \in L^{\infty}$. As a consequence if $\mathrm{W} \in L^{p}$ for some $p \in$ $(1, \infty), \mathrm{W} \in L^{q}$ for all $q \geq p$ : the smaller $p$ is, the more stringent the assumption.

This result turns out to be very useful to prove rigidity theorems. In particular, assuming further that the manifold $X$ is simply connected at infinity forces $(X, g)$ to be isometric to the hyperbolic space (see Theorem 4.1). We also give a variant of this theorem for static spacetimes together with a rigidity result in Section 4.

Einstein metrics constructed in $[7,18,22]$ satisfy $|\mathrm{W}| \leq C \mathrm{e}^{-2 \rho}$. In particular $\mathrm{W} \in L^{p}$ for any $p>\frac{n-1}{2}$. The case $p=\frac{n-1}{2}$ is more delicate and we plan to address it in a future work. Nevertheless Theorem 3.3 shows that 
Theorem 1.2 remains true for $n \geq 5$ and $p \leq \frac{n-1}{2}$ in the important case of conformally compact metrics.

In the ALE case, the curvature behavior at infinity which is the analog of (1.1) is obtained by a Moser iteration where a global Sobolev inequality is involved. However, as mentioned above, in the ALH case such a kind of global Sobolev inequality is not true (see [15] for an illustration of this fact). Hence, we use a variant of the maximum principle to get Estimate (1.1). This is where the assumption $n \geq 7$ appears. It is also interesting to compare Theorem 1.2 with [10, Theorem 0.13] and [5, Theorem 1.5].

Since we are interested in this article only in complete non-compact manifolds whose curvature will be shown to tend to -1 at infinity, we will always use the shorthand "Einstein manifold" to denote manifolds $\left(X^{n}, g\right)$ satisfying

$$
\text { Ric }=-(n-1) g .
$$

Let us describe the main arguments that lead to Theorem 1.2. First, we note that if $\left(X^{n}, g\right)$ is Einstein, its Weyl tensor satisfies the following well known equation:

$$
\triangle W+2(n-1) W+2 \mathcal{Q}(W)=0
$$

where $\triangle$ is the Laplace operator acting on tensors and $\mathcal{Q}$ is a quadratic expression in the Weyl curvature tensor. See, e.g., [3] for a derivation of this formula. Setting

$$
\mathcal{B}_{\alpha \beta \gamma \delta}:=\mathrm{W}_{\alpha \beta}^{\mu \nu} \mathrm{W}_{\mu \gamma \nu \delta}
$$

$\mathcal{Q}$ can be written as follows:

$$
\mathcal{Q}_{\alpha \beta \gamma \delta}:=\mathcal{B}_{\alpha \beta \gamma \delta}+\mathcal{B}_{\alpha \gamma \beta \delta}-\mathcal{B}_{\beta \alpha \gamma \delta}-\mathcal{B}_{\beta \delta \alpha \gamma}
$$

Note that we are using the Einstein summation convention. Due to the $L^{p}$-bound of $W$, we see that $W$ is small near infinity. Hence, intuitively Equation (1.2) is almost equivalent to the following linear equation:

$$
\triangle W+2(n-1) W=0
$$

By some careful analysis, we are able to show an $L^{2}$-spectral estimate of the Laplace operator acting on Weyl-type tensors (see Lemma 2.8). Together with a refined Kato inequality and some other techniques we achieve the proof of Estimate (1.1). 
Some applications of Theorem 1.2 are considered in this paper. Namely, by Theorem 1.2 we are able to show a rigidity theorem for ALHE manifolds with Weyl tensor belonging to $L^{p}$. We also get the curvature behavior of vacuum static spacetimes with a negative cosmological constant. See Theorems 4.1 and 4.2 for more details.

The rest of the paper goes as follows. In Section 2, we get some basic $L^{2}$-estimates for the Weyl tensor. Then we show how these estimates can be converted to pointwise estimates in Section 3. Finally in Section 4, we discuss some applications of Theorem 1.2.

\section{Basic estimates}

The main purpose of this section is to prove Lemma 2.11 which gives a spectral estimate of some $(0,4)$-tensors on asymptotically hyperbolic Einstein (AHE) manifolds with an essential set. It will play an essential role in the proof of the main theorem.

In particular, Lemma 2.11 will be used to show that if $\|\mathrm{W}\|_{L^{p}\left(X^{n}, g\right)}<\infty$ for some $p \in\left(1, \frac{n-1}{2}\right)$, then $\|\mathrm{W}\|_{L^{2}\left(X^{n}, g\right)}<\infty$. Moreover if $n \geq 6$, we have $\left\|\mathrm{e}^{\frac{a}{2} \rho} \mathrm{W}\right\|_{L^{2}\left(X^{n}, g\right)}<\infty$ for some positive $a$. See Proposition 2.13 for more details.

We choose once and for all a complete non-compact Einstein manifold $\left(X^{n}, g\right)$ containing an essential subset $\mathbb{D}$. We first introduce some estimates for a Riccati equation that will be useful for the analysis of the normal curvature equation. Similar results have been obtained in [27, Lemma 2.3]. See also $[2,3,16,20]$.

In all this section, we use Greek letters to denote indices going from 0 to $n-1$ and Latin letters for indices from 1 to $n-1$. Unless otherwise stated, we use the Einstein summation convention. For any $R \geq 0$, we set

$$
\mathbb{D}_{R}:=\left\{x \in X, d_{g}(x, \mathbb{D}) \leq R\right\}
$$

and denote by

$$
\Sigma_{R}=\rho^{-1}(R)
$$

a slice of constant $\rho$. We denote by $S$ the second fundamental form of the level sets $\Sigma_{R}: S=$ Hess $\rho$.

Lemma 2.1. Let $\varepsilon$ be a positive constant. Assume that $f(\rho)$ is a smooth positive function of $\rho>0$ such that $|f(\rho)-1| \leq \varepsilon$. Assume further that $y$ is 
a solution of

$$
y^{\prime}+y^{2}=f
$$

satisfying

$$
y(0)>0 .
$$

Then $y$ is a positive function satisfying

$$
|y-1| \leq \mathrm{e}^{-\rho / 2}|y(0)-1|+\varepsilon .
$$

Proof. We claim that $y>0$. Indeed, if there exists $\rho$ such that $y(\rho) \leq 0$, we can find some $\rho_{0}$ satisfying $y\left(\rho_{0}\right)=0$ and $y(\tau)>0$ for any $\tau \in\left(0, \rho_{0}\right)$. In particular, this implies that $y^{\prime}\left(\rho_{0}\right) \leq 0$. But

$$
0 \geq y^{\prime}\left(\rho_{0}\right)+y^{2}\left(\rho_{0}\right)=f\left(\rho_{0}\right)>0,
$$

which is a contradiction. Next, we set $z=y-1$. The equation satisfied by $y$ implies the following one for $z$ :

$$
\left(z^{2}\right)^{\prime}+2(y+1) z^{2}=2 z(f-1) .
$$

In particular, since we noticed that $y>0$, we have

$$
\left(z^{2}\right)^{\prime}+2 z^{2}<2 z(f-1) \leq z^{2}+(f-1)^{2} .
$$

This inequality can be integrated to yield

$$
|z| \leq \sqrt{z^{2}(0) \mathrm{e}^{-\rho}+\varepsilon^{2}} \leq|z|(0) \mathrm{e}^{-\rho / 2}+\varepsilon .
$$

For simplicity we may use Fermi coordinates $\left(x^{1}, \ldots, x^{n-1}\right)$ on the slices $\Sigma_{\rho}$. We denote $S_{i j}$ the components of the second fundamental form of $\Sigma_{\rho}$ in this coordinate system. It is well known that the following equation holds:

$$
\frac{\partial}{\partial \rho} S_{i}^{j}+S_{k}^{j} S_{i}^{k}=-\mathrm{Rm}^{j}{ }_{0 i 0}
$$

where the index 0 refers to the unit normal direction of $\Sigma_{\rho}$, that is to say $\nabla \rho$.

From Lemma 2.1 and the fact that $\Sigma_{0}=\mathbf{B}$ is striclty convex, i.e., $S>0$ on $T \Sigma_{0}$, combined with standard methods (see, e.g., [25, Chapter 6]), it follows that the second fundamental form $S$ of the level sets $\Sigma_{R}$ is positive definite for any $R>0$. 
Our next task is to prove that the Weyl tensor of $\left(X^{n}, g\right)$ tends uniformly to zero at infinity, see Lemma 2.3. For this we first prove that the injectivity radius of $\left(X^{n}, g\right)$ is bounded from below by a positive constant:

Lemma 2.2. Assume that $\left(X^{n}, g\right)$ contains an essential set $\mathbb{D}$. Then there exists some positive constant $r_{0}>0$ such that the injectivity radius $i n j_{\left(X^{n}, g\right)}$ $(x)>r_{0}$ for any point $x \in\left(X^{n}, g\right)$.

Proof. The injectivity radius of $i n j_{\left(X^{n}, g\right)}(x), x \in X$, is a positive continuous map from $X$ to $\mathbb{R}_{+}^{*} \cup\{\infty\}$. Hence, it is bounded from below on $\mathbb{D}_{2}=\{x \in$ $\left.X, d_{g}(x, \mathbb{D}) \leq 2\right\}$ by some $r_{0}$. We can assume $r_{0}<1$. Since $\sec _{g}<0$ on $X \backslash \mathbb{D}$, for any point $x \in X \backslash \mathbb{D}_{2}$, the exponential map from $x_{0}$ has no critical point at radius smaller than $r_{0}$. Assume that there exists a point $x \in X^{n} \backslash \mathbb{D}_{2}$ whose injectivity radius is less than $\frac{r_{0}}{2}$. We can assume that any point $y$ with $2<\rho(y)<\rho(x)$ has injectivity radius strictly greater than $\frac{r_{0}}{2}$. Then there exists a geodesic $\gamma:[0,1] \rightarrow X$ of length $r_{0}$ such that $\gamma(0)=\gamma(1)=x$. Note that $r_{0}<1$, so the whole geodesic $\gamma$ remains in $X^{n} \backslash \mathbb{D}$ where sectional curvature is negative.

The function $\rho \circ \gamma$ is convex and cannot be constant. Indeed, if $\rho(\gamma(t))>$ $0,(\rho \circ \gamma)^{\prime \prime}(t)=S(\dot{\gamma}, \dot{\gamma}) \geq 0$, with equality iff $\dot{\gamma}$ is colinear to $\nabla \rho$. So $\rho(\gamma(1 / 2))$ $<\rho(x)$. Consider now the geodesics $\gamma_{1}$ and $\gamma_{2}$ defined on the interval $[0,1]$ by

$$
\begin{aligned}
& \gamma_{1}(t)=\gamma\left(\frac{1+t}{2}\right) \\
& \gamma_{2}(t)=\gamma\left(\frac{1-t}{2}\right)
\end{aligned}
$$

They are two geodesics starting at $\gamma(1 / 2)$ and ending at $x$, both of length $\frac{r_{0}}{2}$. This means that $\gamma(1 / 2)$ has injectivity radius less than $\frac{r_{0}}{2}$ and contradicts the definition of $x$.

Lemma 2.3. Assuming further that $\|\mathrm{W}\|_{L^{p}\left(X^{n}, g\right)}<\infty$ for some $p \in(1, \infty)$, the Weyl tensor $\mathrm{W}$ of $\left(X^{n}, g\right)$ tends uniformly to zero at infinity.

Proof. We first remark that the Riemann tensor of $g$ is globally bounded. Indeed, on the one hand, since $\mathbb{D}$ is compact, $|\mathrm{Rm}|^{2}$ is bounded on $\mathbb{D}$. On the other hand, the sectional curvature is negative outside $\mathbb{D}$. As a consequence, for any point $x \in M \backslash \mathbb{D}$, and any 2-plane $\pi \in T_{x} M$, choosing an arbitrary 
orthonormal basis $\left(e_{1}, e_{2}, \ldots, e_{n}\right)$ such that $\pi=\operatorname{vect}\left(e_{1}, e_{2}\right)$, we have

$$
\begin{aligned}
0 \geq \sec _{g}(\pi) & =\operatorname{Rm}\left(e_{1}, e_{2}, e_{1}, e_{2}\right) \geq \sum_{i} \operatorname{Rm}\left(e_{1}, e_{i}, e_{1}, e_{i}\right) \\
& =\operatorname{Ric}\left(e_{1}, e_{1}\right)=-(n-1)
\end{aligned}
$$

so the sectional curvature and the curvature tensor are uniformly bounded on $M \backslash \mathbb{D}$. In particular, the Weyl tensor of $\left(X^{n}, g\right)$ is uniformly bounded. We compute next $\triangle|\mathrm{W}|^{2}$ in two different ways:

$$
\frac{1}{2} \triangle|\mathrm{W}|^{2}=\langle\mathrm{W}, \triangle \mathrm{W}\rangle+\langle\nabla \mathrm{W}, \nabla \mathrm{W}\rangle=|\mathrm{W}| \triangle|\mathrm{W}|+\langle\nabla|\mathrm{W}|, \nabla|\mathrm{W}|\rangle .
$$

From Kato's inequality $|\nabla| \mathrm{W}|| \leq|\nabla \mathrm{W}|$, we infer

$$
\begin{aligned}
|\mathrm{W}| \triangle|\mathrm{W}| & \geq\langle\mathrm{W}, \triangle \mathrm{W}\rangle \\
& \geq-2(n-1)|\mathrm{W}|^{2}-2\langle\mathrm{~W}, \mathcal{Q}(\mathrm{W})\rangle \\
& \geq-2(n-1)|\mathrm{W}|^{2}-2|\mathrm{~W}||\mathcal{Q}(\mathrm{W})| \\
& \geq-2(n-1)|\mathrm{W}|^{2}-8|\mathrm{~W}|^{3}
\end{aligned}
$$

where we used Equation (1.2) and the fact that $|\mathcal{Q}(\mathrm{W})| \leq 4|\mathrm{~W}|^{2}$. In particular, around any point where $|\mathrm{W}| \neq 0$, we have the following differential inequality for the norm of the Weyl tensor:

$$
-\triangle|\mathrm{W}| \leq\left[2(n-1)+8\|\mathrm{~W}\|_{\infty}\right]|\mathrm{W}|
$$

Let $r_{0}$ be a lower bound for the injectivity radius of $\left(X^{n}, g\right)$. From $\mathrm{W} \in$ $L^{p}$, we get that for any small $\mu>0$ there exists $R>r_{0}$ such that

$$
\int_{X^{n} \backslash \mathbb{D}_{R-r_{0}}}|\mathrm{~W}|^{p} d V_{g} \leq \mu .
$$

We select an arbitrary $q>n$ and remark that for any $x_{0} \in X^{n} \backslash \mathbb{D}_{R}$,

$$
\begin{aligned}
\|\mathrm{W}\|_{L^{q}\left(B_{r_{0}}\left(x_{0}\right)\right)} & \leq\|\mathrm{W}\|_{L^{\infty}\left(B_{r_{0}}\left(x_{0}\right)\right)}^{1-\frac{p}{q}}\|\mathrm{~W}\|_{L^{p}\left(B_{r_{0}}\left(x_{0}\right)\right)}^{\frac{p}{q}} \\
& \leq\|\mathrm{W}\|_{L^{\infty}\left(X^{n}\right)}^{1-\frac{p}{q}}\|\mathrm{~W}\|_{L^{p}\left(X^{n} \backslash \mathbb{D}_{R-r_{0}}\right)}^{\frac{p}{q}} \\
& \leq\|\mathrm{W}\|_{L^{\infty}\left(X^{n}\right)}^{1-\frac{p}{q}} \mu^{\frac{1}{q}}
\end{aligned}
$$


It follows from the Moser iteration technique, see, e.g., [17, Theorem 8.17], that there exists a constant $C>0$ depending only on $r_{0}$ such that

$$
\|\mathrm{W}\|_{L^{\infty}\left(B \frac{r_{0}}{2}\left(x_{0}\right)\right)} \leq C \mu^{\frac{1}{q}}
$$

This concludes the proof of the lemma. Note that the coefficients of the metric in normal coordinates are controlled by the $L^{\infty}$-bound on the Riemann tensor.

We define the mean curvature of $\Sigma_{\rho}$ by $H=g^{i j} S_{i j}=S_{i}^{i}$. Since $g$ is Einstein with scalar curvature $-n(n-1)$, the Riemann tensor can be written as follows:

$$
\operatorname{Rm}_{\alpha \beta \gamma \delta}=-\left(g_{\alpha \gamma} g_{\beta \delta}-g_{\alpha \delta} g_{\beta \gamma}\right)+\mathrm{W}_{\alpha \beta \gamma \delta}
$$

Combining Equations (2.1) and (2.3) with Lemma 2.3, we get the following lemma:

Lemma 2.4. Let $H$ be the mean curvature of the hypersurfaces of constant $\rho$. If $\|\mathrm{W}\|_{L^{p}\left(X^{n}, g\right)}<\infty$ for some $p \in(1, \infty)$, then $H=(n-1)+o(1)$.

Proof. We fix an arbitrary $\varepsilon>0$. From Equation (2.3), the Riccati equation for the Weingarten operator (2.1) can be rewritten as follows:

$$
\frac{\partial}{\partial \rho} S_{i}^{j}+S_{k}^{j} S_{i}^{k}=\delta_{i}^{j}-\mathrm{W}^{j}{ }_{0 i 0} .
$$

From Lemma 2.3, there exists $\rho_{0}>0$ such that $|\mathrm{W}|<\varepsilon$ on $X \backslash \mathbb{D}_{\rho_{0}}$. It follows from Lemma 2.1 that $S$ satisfies

$$
|S-\delta| \leq\left(\sup _{\Sigma_{\rho_{0}}}|S-\delta|\right) \mathrm{e}^{-\left(\rho-\rho_{0}\right) / 2}+\varepsilon
$$

on $X^{n} \backslash \mathbb{D}_{\rho_{0}}$. In particular, $H=\operatorname{tr}(S)$ is controlled at infinity:

$$
|H-(n-1)| \leq(n-1)\left(\sup _{\Sigma_{\rho_{0}}}|S-\delta|\right) \mathrm{e}^{-\left(\rho-\rho_{0}\right) / 2}+(n-1) \varepsilon .
$$

Since $\varepsilon$ was arbitrary, this proves the lemma. 
As a consequence of this lemma, we get the following $L^{2}$-estimate:

Lemma 2.5 (Cheng-Yau estimate). Assume that $\|\mathrm{W}\|_{L^{p}\left(X^{n}, g\right)}<\infty$ for some $p \in(1, \infty)$. For every $\varepsilon>0$, there exists a compact subset $K_{\varepsilon} \supset \mathbb{D}$ such that for any $u \in C_{c}^{\infty}\left(X \backslash K_{\varepsilon}\right)$,

$$
-\int_{X} u \triangle u d V_{g} \geq\left[\frac{(n-1)^{2}}{4}-\varepsilon\right] \int_{X} u^{2} d V_{g}
$$

Proof. We set $\varphi=\mathrm{e}^{-\frac{n-1}{2} \rho}$. Remark that if $\rho_{0}$ is large enough, $H \geq(n-1)-$ $\frac{2 \varepsilon}{n-1}$ on $X \backslash \mathbb{D}_{\rho_{0}}$ :

$$
-\triangle \varphi=-\frac{(n-1)^{2}}{4} \varphi+\frac{n-1}{2} H \varphi \geq\left(\frac{(n-1)^{2}}{4}-\varepsilon\right) \varphi .
$$

We rewrite

$$
\begin{aligned}
\triangle u & =\triangle\left(\varphi \frac{u}{\varphi}\right) \\
& =\frac{\triangle \varphi}{\varphi} u+2\left\langle d \varphi, d \frac{u}{\varphi}\right\rangle+\varphi \triangle \frac{u}{\varphi} .
\end{aligned}
$$

So,

$$
\begin{aligned}
-\int_{X \backslash \mathbb{D}_{\rho_{0}}} u \triangle u d V_{g}= & -\int_{X \backslash \mathbb{D}_{\rho_{0}}} \frac{\triangle \varphi}{\varphi} u^{2} d V_{g}-2 \int_{X \backslash \mathbb{D}_{\rho_{0}}} u\left\langle d \varphi, d \frac{u}{\varphi}\right\rangle d V_{g} \\
& -\int_{X \backslash \mathbb{D}_{\rho_{0}}} u \varphi \triangle \frac{u}{\varphi} d V_{g} \\
\geq & \left(\frac{(n-1)^{2}}{4}-\varepsilon\right) \int_{X \backslash \mathbb{D}_{\rho_{0}}} u^{2} d V_{g} \\
& -2 \int_{X \backslash \mathbb{D}_{\rho_{0}}} u\left\langle d \varphi, d \frac{u}{\varphi}\right\rangle d V_{g} \\
& +\int_{X \backslash \mathbb{D}_{\rho_{0}}}\left\langle d(u \varphi), d \frac{u}{\varphi}\right\rangle d V_{g} \\
\geq & \left(\frac{(n-1)^{2}}{4}-\varepsilon\right) \int_{X \backslash \mathbb{D}_{\rho_{0}}} u^{2} d V_{g} \\
& -\int_{X \backslash \mathbb{D}_{\rho_{0}}} u\left\langle d \varphi, d \frac{u}{\varphi}\right\rangle d V_{g}
\end{aligned}
$$




$$
\begin{gathered}
\quad+\int_{X \backslash \mathbb{D}_{\rho_{0}}} \varphi\left\langle d u, d \frac{u}{\varphi}\right\rangle d V_{g} \\
\geq\left(\frac{(n-1)^{2}}{4}-\varepsilon\right) \int_{X \backslash \mathbb{D}_{\rho_{0}}} u^{2} d V_{g} \\
\quad+\int_{X \backslash \mathbb{D}_{\rho_{0}}} \varphi^{2}\left|d \frac{u}{\varphi}\right|^{2} d V_{g} \\
\geq\left(\frac{(n-1)^{2}}{4}-\varepsilon\right) \int_{X \backslash \mathbb{D}_{\rho_{0}}} u^{2} d V_{g} .
\end{gathered}
$$

As noted in $[18,22]$, this simple estimate immediately yields an estimate for the covariant Laplacian acting on tensor fields by making use of Kato's inequality. Unfortunately, this estimate is not sharp enough to get useful estimates. In [22], Lee mainly deals with symmetric 2-tensors. In order to get sharp estimates he considers $r$-tensor fields as $(r-1)$-tensor-valued 1 -forms. However, our interest is in tensors which can be seen as $\Lambda^{2} X$-valued 2 -forms. We recall here some results on these forms. We refer the reader to [6, Chapter 1], [22, Chapter 7] and references therein for more details.

Definition 2.6. We say that a $(0, p+2)$-tensor $\omega$ belongs to $\Lambda^{p} T^{* 2} X$, if it satisfies

$$
\begin{aligned}
& \omega\left(Y_{1}, Y_{2} ; Z_{1}, \ldots, Z_{s}, \ldots, Z_{l}, \ldots, Z_{p}\right) \\
& =-\omega\left(Y_{1}, Y_{2} ; Z_{1}, \ldots, Z_{s-1}, Z_{l}, Z_{s+1}, \ldots, Z_{l-1}, Z_{s}, Z_{l+1}, \ldots, Z_{p}\right),
\end{aligned}
$$

for every $Y_{1}, Y_{2}, Z_{1}, \ldots, Z_{p} \in T X$ and any pair $s, l$ with $1 \leq s<l \leq p$.

In local coordinates this translates to

$$
\omega_{\mu \nu \alpha_{1} \ldots \alpha_{l} \ldots \alpha_{s} \ldots \alpha_{p}}=-\omega_{\mu \nu \alpha_{1} \ldots \alpha_{s} \ldots \alpha_{l} \ldots \alpha_{p}} \quad(1 \leq s<l \leq p) .
$$

For any local orthogonal frame $\left\{e_{\mu}\right\}$ and dual coframe $\left\{e^{\mu}\right\}$, the exterior derivative

$$
D: C^{\infty}\left(X ; \Lambda^{p} T^{* 2} X\right) \rightarrow C^{\infty}\left(X ; \Lambda^{p+1} T^{* 2} X\right)
$$

on $T^{* 2} X$-valued $p$-forms is given by

$$
D \omega:=e^{\mu} \wedge \nabla_{e_{\mu}} \omega
$$

for every $\omega \in \Lambda^{p} T^{* 2} X$. It is standard matter to check that $D$ does not depend on the choice of the frame $\left\{e^{\mu}\right\}$. 
Let $D^{*}$ be the formal $L^{2}$-adjoint of $D$. If $\omega \in \Lambda^{p} T^{* 2} X$, we define the divergence of $\omega, \operatorname{div} \omega \in \Lambda^{p-1} T^{* 2} X$, as follows:

$$
\operatorname{div} \omega\left(X_{1}, X_{2} ; Y_{1}, \ldots, Y_{p-1}\right):=\sum_{m=1}^{n}\left(\nabla_{e_{m}} \omega\right)\left(X_{1}, X_{2} ; e_{m}, Y_{1}, \ldots, Y_{p-1}\right)
$$

It can be shown that $D^{*}=-$ div.

We define the Hodge Laplacian on $T^{* 2} X$-valued $p$-forms $\Lambda^{p} T^{* 2} X$ as follows

$$
\widetilde{\triangle}:=D D^{*}+D^{*} D
$$

and the covariant Laplace operator on $\omega \in \Lambda^{p} T^{* 2} X$ by

$$
\triangle \omega=\operatorname{tr}\left(\nabla^{2} \omega\right)
$$

where the trace is taken with respect to the two indices of the Hessian.

For a 1-form $\theta \in T^{*} X$, we let $\theta \vee: \Lambda^{p} T^{* 2} X \rightarrow \Lambda^{p-1} T^{* 2} X$ denote the adjoint of the map $\theta \wedge: \Lambda^{p-1} T^{* 2} X \rightarrow \Lambda^{p} T^{* 2} X$ with respect to $g$, so that $\langle\theta \wedge \omega, \eta\rangle=\langle\omega, \theta \vee \eta\rangle$ for $\omega \in \Lambda^{p} T^{* 2} X$ and $\eta \in \Lambda^{p+1} T^{* 2} X$. In coordinates,

$$
(\theta \vee \omega)_{\mu \nu ; \alpha_{1} \ldots \alpha_{p-1}}=g^{\gamma \delta} \theta_{\gamma} \omega_{\mu \nu ; \delta \alpha_{1} \ldots \alpha_{p-1}}
$$

For any $\xi \in \Lambda^{p} T^{* 2} X$ and any function $u$, we define $H(u) \xi$ as

$$
H(u) \xi:=\left(\nabla_{e_{\mu}, e_{\nu}}^{2} u\right) e^{\mu} \wedge\left(e^{\nu} \vee \xi\right)
$$

Given $\omega \in \Lambda^{p} T^{2} X$ and a function $f$, we have the following formulas:

1. $D(f \omega)=f D \omega+d f \wedge \omega$,

2. $D^{*}(f \omega)=f D^{*} \omega-d f \vee \omega$,

3. $D^{*}(d f \wedge \omega)=-(\triangle f) \omega-\nabla_{\nabla_{f}} \omega-d f \wedge D^{*} \omega+H(f) \omega$,

4. $|d f \wedge \omega|^{2}+|d f \vee \omega|^{2}=|d f|^{2}|\omega|^{2}$.

The following lemma is taken from [22, Lemma 7.9] (see also [12]):

Lemma 2.7. For any smooth compactly supported section $\xi$ of $\Lambda^{q} T^{* 2} X$, and any positive $C^{2}$ function $\varphi$ on $X$, the following integral formula holds

$$
(\xi, \widetilde{\triangle} \xi) \geq \int_{X}\left\langle\xi,\left(-\varphi^{-1} \triangle \varphi+2 H(\log \varphi) \xi\right)\right\rangle d V_{g}
$$


Here $\langle\cdot, \cdot\rangle$ is the induced inner product of tensor bundles and $(\cdot, \cdot)$ is $\int_{X^{n}}\langle\cdot, \cdot\rangle d V_{g}$.

As in [22, Lemma 7.10] and [23, Lemma 2.2], we also have the following result:

Lemma 2.8. Let $\left(X^{n}, g\right)$ be a complete non-compact Einstein manifold of dimension $n \geq 6$. Then for every small $\varepsilon>0$ there exists a compact set $K_{1}(\varepsilon)$ such that the following estimate holds for any smooth section $\xi$ of $\Lambda^{2} T^{* 2} X$ compactly supported in $X^{n} \backslash K_{1}(\varepsilon)$ :

$$
(\xi, \widetilde{\triangle} \xi) \geq\left[\frac{(n-5)^{2}}{4}-\varepsilon\right] \int_{X^{n}}|\xi|^{2} d V_{g}
$$

Proof. We let $\left\{e^{\mu}\right\}, 0 \leq \mu \leq n-1$ be a local orthonormal coframe of $g$ such that $e_{0}=d \rho$. This implies that $\left\{e_{i}\right\}, 1 \leq i \leq n-1$ is tangent to $\Sigma_{\rho}$. For convenience, we also denote $g=d \rho^{2}+g_{i j}(\rho, \theta) d x^{i} d x^{j}$. We denote $\varepsilon^{\prime}:=\frac{\varepsilon}{3 n-11}$. We set

$$
\varphi_{2}(x)=\mathrm{e}^{-\frac{n-5}{2} \rho} .
$$

Arguing as in the proof of Lemma 2.4, we get that if $\rho_{0}$ is large enough,

$$
|S-\delta| \leq \varepsilon^{\prime}
$$

on $X \backslash \mathbb{D}_{\rho_{0}}$. Restricting ourselves to $X \backslash \mathbb{D}_{\rho_{0}}$, this implies that

$$
\begin{aligned}
-\varphi_{2}^{-1} \triangle \varphi_{2} & =-\frac{(n-5)^{2}}{4}+\frac{n-5}{2} \triangle \rho \\
& \geq-\frac{(n-5)^{2}}{4}+\frac{(n-5)(n-1)}{2}-(n-1) \varepsilon^{\prime} \\
& =\frac{(n-5)(n+3)}{4}-(n-1) \varepsilon^{\prime},
\end{aligned}
$$

and

$$
\begin{aligned}
\nabla_{i, j}^{2} \log \varphi_{2} & =-\frac{n-5}{2} \nabla_{i, j}^{2} \rho \\
& \geq-\frac{n-5}{2} g_{i j}-\varepsilon^{\prime} g_{i j} \\
\nabla_{0, j}^{2} \log \varphi_{2} & =0 \\
\nabla_{0,0}^{2}\left(\log \varphi_{2}\right) & =0
\end{aligned}
$$


From these estimates, we get that, for any $\xi$ which is compactly supported in $X \backslash \mathbb{D}_{\rho_{0}}$,

$$
\begin{aligned}
\left\langle 2 H\left(\log \varphi_{2}\right) \xi, \xi\right\rangle & =\left\langle 2 \nabla_{\mu, \nu}^{2}\left(\log \varphi_{2}\right) \mathrm{e}^{\mu} \wedge\left(e^{\nu} \vee \xi\right), \xi\right\rangle \\
& =\nabla_{\mu, \nu}^{2}\left(\log \varphi_{2}\right)\left\langle e^{\mu} \vee \xi, e^{\nu} \vee \xi\right\rangle \\
& =2 \nabla_{e^{i}, e^{j}}^{2}\left(\log \varphi_{2}\right)\left\langle e^{i} \vee \xi, e^{j} \vee \xi\right\rangle \\
& \geq-2\left(\frac{n-5}{2}+\frac{n-5}{2} \varepsilon^{\prime}\right) \delta_{i j}\left\langle e^{i} \vee \xi, e^{j} \vee \xi\right\rangle \\
& \geq-\left(2(n-5)+(n-5) \varepsilon^{\prime}\right)\langle\xi, \xi\rangle
\end{aligned}
$$

Here we have used the fact that

$$
\sum_{i=1}^{n-1}\left|e^{i} \vee \xi\right|^{2} \leq \sum_{\mu=0}^{n-1}\left|e^{\mu} \vee \xi\right|^{2}=2|\xi|^{2}
$$

for $\xi \in \Lambda^{2} T^{2} X$. Combining Equation (2.6) and (2.5) and Lemma 2.7, we have

$$
\begin{aligned}
(\xi, \widetilde{\triangle} \xi) & \geq \int_{X^{n}}\left(\frac{(n-5)(n+3)}{4}-2(n-5)-(3 n-11) \varepsilon^{\prime}\right)\langle\xi, \xi\rangle d V_{g} \\
& \geq\left(\frac{(n-5)^{2}}{4}-\varepsilon\right) \int_{X^{n}}|\xi|^{2} d V_{g} .
\end{aligned}
$$

This proves the lemma with $K_{1}(\varepsilon)=\mathbb{D}_{\rho_{0}}$.

Note that a $(0,4)$-tensor $\omega$ such that $\omega\left(\cdot, \cdot ; Y_{1}, Y_{2}\right)=-\omega\left(\cdot, \cdot ; Y_{2}, Y_{1}\right)$ for any $Y_{1}, Y_{2} \in T X$, can be considered as a $\Lambda^{2} X$-valued 2 -form, i.e., $\omega \in \Lambda^{2}$ $\left(X, \Lambda^{2} X\right)$. In the remaining of this section we will consider such $(0,4)$-tensors. The following lemma gives a Weitzenböck formula relating the covariant Laplacian on such tensors to $\widetilde{\triangle}$ (see $[8$, Proposition 4.1$]$ for a more general formula):

Lemma 2.9. For a section $\omega$ of $\Lambda^{2}\left(X, \Lambda^{2} X\right)$,

$$
\begin{aligned}
\widetilde{\triangle} \omega_{\alpha \beta \gamma \delta}= & -\triangle \omega_{\alpha \beta \gamma \delta}+\operatorname{Ric}_{\gamma}^{\nu} \omega_{\alpha \beta \nu \delta}-\operatorname{Ric}_{\delta}{ }_{\delta} \omega_{\alpha \beta \nu \gamma} \\
& -\operatorname{Rm}_{\delta}{ }^{\nu}{ }_{\gamma}^{\mu} \omega_{\alpha \beta \mu \nu}+\operatorname{Rm}^{\nu}{ }_{\alpha \gamma}{ }^{\mu} \omega_{\nu \beta \mu \delta}+\operatorname{Rm}_{\beta \gamma}{ }_{\beta \gamma}{ }^{\mu} \omega_{\alpha \nu \mu \delta} \\
& -\operatorname{Rm}_{\gamma}{ }^{\nu \mu}{ }_{\delta} \omega_{\alpha \beta \mu \nu}+\operatorname{Rm}_{\alpha}^{\nu}{ }_{\alpha}{ }^{\mu} \omega_{\nu \beta \mu \gamma}+\operatorname{Rm}_{\beta}{ }_{\beta}{ }^{\mu}{ }_{\delta} \omega_{\alpha \nu \mu \gamma} .
\end{aligned}
$$


Proof. Note that the last two indices of $\omega$ are considered to be the 2 -form indices. By some direct computations, we get

$$
\begin{aligned}
\left(D^{*} D \omega\right)_{\alpha \beta \gamma \delta} & =-\nabla^{\mu}(D \omega)_{\alpha \beta \mu \gamma \delta} \\
& =-\nabla^{\mu} \nabla_{\mu} \omega_{\alpha \beta \gamma \delta}+\nabla^{\mu} \nabla_{\gamma} \omega_{\alpha \beta \mu \delta}-\nabla^{\mu} \nabla_{\delta} \omega_{\alpha \beta \mu \gamma} \\
\left(D D^{*} \omega\right)_{\alpha \beta \gamma \delta} & =\left(D^{*} \omega_{\alpha \beta \delta}\right)_{\gamma}-\left(D^{*} \omega_{\alpha \beta \gamma}\right)_{\delta} \\
& =-\nabla_{\gamma} \nabla^{\mu} \omega_{\alpha \beta \mu \delta}+\nabla_{\delta} \nabla^{\mu} \omega_{\alpha \beta \mu \gamma} .
\end{aligned}
$$

Then, applying the Ricci identity, we finally get

$$
\begin{aligned}
\widetilde{\triangle} \omega_{\alpha \beta \gamma \delta}= & \left.D D^{*}+D^{*} D\right) \omega_{\alpha \beta \gamma \delta} \\
= & -\nabla^{\mu} \nabla_{\mu} \omega_{\alpha \beta \gamma \sigma}+\nabla^{\mu} \nabla_{\gamma} \omega_{\alpha \beta \mu \sigma}-\nabla_{\gamma} \nabla^{\mu} \omega_{\alpha \beta \mu \sigma}{ }^{\mu} \\
& \quad+\nabla_{\sigma} \nabla^{\mu} \omega_{\alpha \beta \mu \gamma}-\nabla^{\mu} \nabla_{\sigma} \omega_{\alpha \beta \mu \gamma} \\
= & -\triangle \omega_{\alpha \beta \gamma \sigma}+\operatorname{Ric}_{\gamma}{ }_{\gamma} \omega_{\alpha \beta \nu \sigma}+\operatorname{Rm}_{\delta \gamma}^{\nu}{ }^{\mu} \omega_{\alpha \beta \mu \nu}-\operatorname{Rm}_{\alpha}{ }^{\nu}{ }_{\gamma}{ }^{\mu} \omega_{\nu \beta \mu \sigma} \\
& \quad-\operatorname{Rm}_{\beta}{ }^{\nu}{ }_{\gamma}{ }^{\mu} \omega_{\alpha \nu \mu \sigma} \\
- & \operatorname{Ric}_{\delta}{ }_{\delta} \omega_{\alpha \beta \nu \gamma}+\operatorname{Rm}_{\gamma}{ }_{\gamma}{ }^{\mu}{ }_{\delta} \omega_{\alpha \beta \mu \nu}-\operatorname{Rm}_{\alpha}{ }^{\nu \mu}{ }_{\delta} \omega_{\nu \beta \mu \gamma}-\operatorname{Rm}_{\beta}{ }^{\nu \mu}{ }_{\delta} \omega_{\alpha \nu \mu \gamma} .
\end{aligned}
$$

Definition 2.10. We say that a 4-tensor $\omega$ belongs to $\widetilde{\Sigma}^{4}$ if it satisfies the following three assumptions:

1. $\omega_{\alpha \beta \gamma \delta}=-\omega_{\beta \alpha \gamma \delta}$,

2. $\omega_{\alpha \beta \gamma \delta}+\omega_{\alpha \gamma \delta \beta}+\omega_{\alpha \delta \beta \gamma}=0$,

3. $\omega_{\alpha \beta \gamma \delta}=\omega_{\gamma \delta \alpha \beta}$.

Furthermore, if $\omega$ is trace-free, meaning that $g^{i k} \omega_{i j k l}=0$, we say that $\omega \in \widetilde{\Sigma}_{0}^{4}$.

Note that any element of $\widetilde{\Sigma}^{4}$ belongs to $\Lambda^{2}\left(X, \Lambda^{2} X\right)$. Combining Lemmas 2.8 and 2.9, we obtain the following estimate:

Lemma 2.11. Let $\left(X^{n}, g\right)$ be an $n$-dimensional Einstein manifold containing an essential set $\mathbb{D}$ with $n>5$. Then for every $\varepsilon>0$ there exists a compact set $K_{2}(\varepsilon) \supset \mathbb{D}$ such that the following estimate holds for any smooth 4-tensor $\omega \in \widetilde{\Sigma}_{0}^{4}$ compactly supported in $X^{n} \backslash K_{2}(\varepsilon)$ :

$$
\int_{X^{n}}|\nabla \omega|^{2} d V_{g} \geq\left(\frac{(n-1)^{2}}{4}+4-C(n, \varepsilon)\right) \int_{X^{n}}|\omega|^{2} d V_{g} .
$$


Here we denote by $C(n, \varepsilon)$ some constant depending only on $n, \varepsilon$ and

$$
\lim _{\varepsilon \rightarrow 0} C(n, \varepsilon)=0
$$

Proof. From Lemma 2.3, there exists a compact set $K_{2}(\varepsilon) \supset \mathbb{D}$ such that

$$
\|\mathrm{Rm}-\mathbf{K}\|_{L^{\infty}\left(X^{n} \backslash K_{2}(\varepsilon)\right)}=\|\mathrm{W}\|_{L^{\infty}\left(X^{n} \backslash K_{2}(\varepsilon)\right)} \leq \varepsilon .
$$

By a direct computation, we have

$$
\begin{aligned}
& \operatorname{Ric}_{\gamma}^{\nu} \omega_{\alpha \beta \nu \delta}-\operatorname{Ric}_{\delta}^{\nu} \omega_{\alpha \beta \nu \gamma}=-2(n-1) \omega_{\alpha \beta \gamma \delta} ; \\
& -\operatorname{Rm}_{\delta}{ }^{\nu}{ }_{\gamma}{ }^{\mu} \omega_{\alpha \beta \mu \nu}+\operatorname{Rm}^{\nu}{ }_{\alpha \gamma}{ }^{\mu} \omega_{\nu \beta \mu \delta}+\operatorname{Rm}_{\beta \gamma}{ }_{\beta \gamma}{ }^{\mu} \omega_{\alpha \nu \mu \delta} \\
& \quad=-\omega_{\alpha \beta \delta \gamma}-\omega_{\gamma \beta \alpha \delta}-\omega_{\alpha \gamma \beta \delta}+O(\varepsilon \omega) \\
& \quad=O(\varepsilon \omega) ; \\
& -\operatorname{Rm}_{\gamma}{ }^{\nu \mu}{ }_{\delta} \omega_{\alpha \beta \mu \nu}+\operatorname{Rm}^{\nu}{ }_{\alpha}{ }_{\alpha}{ }_{\delta} \omega_{\nu \beta \mu \gamma}+\operatorname{Rm}^{\nu}{ }_{\beta}{ }^{\mu}{ }_{\delta} \omega_{\alpha \nu \mu \gamma} \\
& \quad=\omega_{\alpha \beta \gamma \delta}+\omega_{\delta \beta \alpha \gamma}+\omega_{\alpha \delta \beta \gamma}+O(\varepsilon \omega) \\
& =O(\varepsilon \omega) .
\end{aligned}
$$

Using Lemma 2.9 together with Lemma 2.8, we get:

$$
\begin{aligned}
\int_{X^{n}} & |\nabla \omega|^{2} d V_{g}=(\omega,-\triangle \omega) \\
= & \left(\omega, \widetilde{\triangle} \omega_{\alpha \beta \gamma \delta}-\operatorname{Ric}_{\gamma}^{\nu} \omega_{\alpha \beta \nu \delta}+\operatorname{Ric}_{\delta}^{\nu} \omega_{\alpha \beta \nu \gamma}\right. \\
& +\operatorname{Rm}_{\delta}^{\nu}{ }_{\gamma}{ }^{\mu} \omega_{\alpha \beta \mu \nu}-\operatorname{Rm}_{\alpha \gamma}^{\nu}{ }^{\mu} \omega_{\nu \beta \mu \delta} \\
& -\operatorname{Rm}_{\beta \gamma}^{\nu}{ }^{\mu} \omega_{\alpha \nu \mu \delta}+\operatorname{Rm}_{\gamma}{ }^{\nu \mu}{ }_{\delta} \omega_{\alpha \beta \mu \nu} \\
& \left.-\operatorname{Rm}_{\alpha}^{\nu}{ }_{\alpha}{ }_{\delta} \omega_{\nu \beta \mu \gamma}-\operatorname{Rm}_{\beta}^{\nu}{ }_{\beta}{ }_{\delta} \omega_{\alpha \nu \mu \gamma}\right) \\
\geq & \frac{(n-5)^{2}}{4} \int_{X^{n}}|\omega|^{2} d V_{g}+2(n-1) \int_{X^{n}}|\omega|^{2} d V_{g}-C(n, \varepsilon) \int_{X^{n}}|\omega|^{2} d V_{g} \\
\geq & \left(\frac{(n-1)^{2}}{4}+4-C(n, \varepsilon)\right) \int_{X^{n}}|\omega|^{2} d V_{g} .
\end{aligned}
$$

Remark 2.12. By a density argument, it is not difficult to see that Lemmas 2.5 and 2.11 are still true if we replace the condition that $u$ or $\omega$ has compact support by $u \in W_{0}^{1,2}\left(X^{n} \backslash K_{1}\right)$ (resp. $\omega \in W_{0}^{1,2}\left(X^{n} \backslash K_{2}\right)$ ). Here the subscript 0 means that $u$ (resp. $\omega$ ) has vanishing trace on $\partial K_{1}$ $\left(\right.$ resp. $\left.\partial K_{2}\right)$. 
Our next goal is to make use of the above estimates to get weighted $L^{2}$-estimate for the Weyl tensor. More precisely, we have the following Agmon type estimate:

Proposition 2.13. Suppose that $\left(X^{n}, g\right), n \geq 4$, is a complete non-compact Einstein manifold with an essential set $\mathbb{D}$. If $\|\mathrm{W}\|_{L^{p}\left(X^{n}, g\right)}<\infty$ for some $1<p<\frac{n-1}{2}$, then

$$
\|\mathrm{W}\|_{L^{2}\left(X^{n}, g\right)}<\infty .
$$

Furthermore if $n \geq 6$, we have $\left\|\mathrm{e}^{\frac{a}{2} \rho} \mathrm{W}\right\|_{W^{1,2}\left(X^{n}, g\right)}<\infty$ for any $a \in[0, n-5)$.

A similar estimate was proven in [24, Theorem 2.1] for functions. While it could be straightforwardly extended to tensors, we choose to give here a simpler derivation in our context. Before giving the proof of this proposition, we need to make a preliminary definition. Formula (1.4) together with Equation (1.3) define a quadratic map from $\widetilde{\Sigma}^{4}$ to itself. We define the associated symmetric bilinear map as follows:

$$
\mathcal{Q}(\xi, \omega)_{\alpha \beta \gamma \delta}:=\xi_{\alpha \beta}^{\mu \nu} \omega_{\mu \gamma \nu \delta}+\xi_{\alpha \gamma}^{\mu \nu} \omega_{\mu \beta \nu \delta}-\xi_{\beta \alpha}^{\mu \nu} \omega_{\mu \gamma \nu \delta}-\xi_{\beta}^{\mu \nu}{ }_{\beta} \omega_{\mu \alpha \nu \gamma}
$$

This map enjoys the following nice property:

Claim 2.14. For every $\omega, \xi \in \widetilde{\Sigma}^{4}$, we have

$$
\langle\mathcal{Q}(\omega, \mathrm{W}), \xi\rangle=\langle\omega, \mathcal{Q}(\xi, \mathrm{W})\rangle
$$

Equivalently, the map $\omega \mapsto \mathcal{Q}(\omega, \mathrm{W})$ is symmetric.

Proof. The proof is a straightforward calculation:

$$
\begin{aligned}
& \langle\mathcal{Q}(\omega, \mathrm{W}), \xi\rangle \\
& =\left(\omega_{\alpha \beta}^{\mu \nu} \mathrm{W}_{\mu \gamma \nu \delta}+\omega_{\alpha \gamma}^{\mu \nu} \mathrm{W}_{\mu \beta \nu \delta}-\omega_{\beta \alpha}^{\mu{ }^{\nu} \mathrm{W}_{\mu \gamma \nu \delta}}-\omega_{\beta \delta}^{\mu{ }^{\nu} \mathrm{W}_{\mu \alpha \nu \gamma}}\right) \xi^{\alpha \beta \gamma \delta} \\
& =2\left(\omega_{\alpha \beta}^{\mu \nu} \mathrm{W}_{\mu \gamma \nu \delta}+\omega_{\alpha \gamma}^{\mu \nu} \mathrm{W}_{\mu \beta \nu \delta}\right) \xi^{\alpha \beta \gamma \delta} \\
& =2 \omega^{\mu \nu}{ }_{\alpha \beta} \mathrm{W}_{\mu \gamma \nu \delta}\left(\xi^{\alpha \beta \gamma \delta}+\xi^{\alpha \gamma \beta \delta}\right) \\
& =-2 \omega_{\alpha \beta}^{\mu \nu} \mathrm{W}_{\mu \gamma \nu \delta}\left(2 \xi^{\alpha \gamma \delta \beta}+\xi^{\alpha \delta \beta \gamma}\right) \\
& =4 \omega_{\alpha \mu \beta \nu} \xi^{\alpha \gamma \beta \delta} \mathrm{W}_{\gamma \delta}^{\mu \nu}-2 \omega_{\alpha \mu \beta \nu} \xi^{\alpha \delta \beta \gamma} \mathrm{W}_{\gamma \delta}^{\mu \nu} \text {. }
\end{aligned}
$$


where we used the first Bianchi identity, Property 2 of Definition 2.10, to get the fourth line. Under this form, the claim becomes clear by swapping $\gamma($ resp. $\delta)$ and $\mu$ (resp. $\nu$ ).

Having made this definition, we can give a proof of Proposition 2.13:

Proof of Proposition 2.13. We remark that if $n<6, p<\frac{n-1}{2} \leq 2$. Hence, from the fact that $\mathrm{W} \in L^{\infty}$, which was proven in Lemma 2.3 , we conclude that $\mathrm{W} \in L^{2}$. As a consequence, we now restrict our attention to the case $n \geq 6$. We also assume that $p>2$. For an arbitrary $b \in \mathbb{R}$ and using Hölder inequality, we get

$$
\int_{X^{n}} \mathrm{e}^{-2 b \rho}|\mathrm{W}|_{g}^{2} d V_{g} \leq\left(\int_{X^{n}}|\mathrm{~W}|_{g}^{p} d V_{g}\right)^{\frac{2}{p}}\left(\int_{X^{n}} \mathrm{e}^{-\frac{2 b p}{p-2} \rho} d V_{g}\right)^{\frac{p-2}{p}} .
$$

As a consequence of the Bishop-Gromov theorem, the second integral appearing in the right-hand side converges if and only if $n-1-\frac{2 b p}{p-2}<0$, that is to say $b>\frac{n-1}{2}-\frac{n-1}{p}$.

From Equation (2.7), we conclude that

$$
\int_{X^{n}} \mathrm{e}^{-2 b \rho}|\mathrm{W}|_{g}^{2} d V_{g}<\infty
$$

for any $b>\frac{n-1}{2}-\frac{n-1}{p}$.

We now select $\varepsilon>0$ to be fixed later and a cutoff function $\chi$ which vanishes on $K_{2}=K_{2}(\varepsilon)$ and which equals one outside a larger compact subset $K_{2}^{\prime} \supset K_{2}(\varepsilon)$. We set $\widetilde{W}:=\chi W$. We remark that $\widetilde{W}$ satisfies the following equation:

$$
\triangle \widetilde{W}+2(n-1) \widetilde{W}+2 \mathcal{Q}(\mathrm{W}, \widetilde{W})=\theta,
$$

where $\theta$ is a tensor belonging to $\widetilde{\Sigma}_{0}^{4}$ and whose support is contained in $\operatorname{supp}(\nabla \chi) \subset K_{2}^{\prime} \backslash K_{2}$.

For any compactly supported Lipschitz function $f$, we have

$$
\begin{aligned}
\int_{X}|\nabla(f \widetilde{W})|^{2} d V_{g} \\
\quad=\int_{X} f^{2}|\nabla \widetilde{W}|^{2} d V_{g}+2 \int_{X} f\langle\nabla f \otimes \widetilde{W}, \nabla \widetilde{W}\rangle d V_{g}+\int_{X}|\nabla f|^{2}|\widetilde{W}|^{2} d V_{g} \\
=\int_{X}\left\langle\nabla\left(f^{2} \widetilde{W}\right), \nabla \widetilde{W}\right\rangle d V_{g}+\int_{X}|\nabla f|^{2}|\widetilde{W}|^{2} d V_{g} \\
=-\int_{X} f^{2}\langle\widetilde{W}, \triangle \widetilde{W}\rangle d V_{g}+\int_{X}|\nabla f|^{2}|\widetilde{W}|^{2} d V_{g}
\end{aligned}
$$




$$
\begin{aligned}
& =2(n-1) \int_{X} f^{2}|\widetilde{W}|^{2} d V_{g}+2 \int_{X} f^{2}\langle\widetilde{W}, \mathcal{Q}(\mathrm{W}, \widetilde{W})\rangle d V_{g} \\
& -\int_{X} f^{2}\langle\widetilde{W}, \theta\rangle d V_{g}+\int_{X}|\nabla f|^{2}|\widetilde{W}|^{2} d V_{g} .
\end{aligned}
$$

Since $f \widetilde{W}$ is compactly supported in $X \backslash K_{2}$, we deduce from Lemma 2.11 that

$$
\begin{aligned}
& {\left[\frac{(n-1)^{2}}{4}+4-\varepsilon\right] \int_{X} f^{2}|\widetilde{W}|^{2} d V_{g}} \\
& \quad \leq 2(n-1) \int_{X} f^{2}|\widetilde{W}|^{2} d V_{g}+2 \int_{X} f^{2}\langle\widetilde{W}, \mathcal{Q}(\mathrm{W}, \widetilde{W})\rangle d V_{g} \\
& \quad-\int_{X} f^{2}\langle\widetilde{W}, \theta\rangle d V_{g}+\int_{X}|\nabla f|^{2}|\widetilde{W}|^{2} d V_{g} .
\end{aligned}
$$

From the fact that $|\mathrm{W}|<\varepsilon$ on $X^{n} \backslash K_{2}$, we conclude that

$$
\begin{aligned}
& {\left[\frac{(n-5)^{2}}{4}-C(n, \varepsilon)\right] \int_{X} f^{2}|\widetilde{W}|^{2} d V_{g}} \\
& \quad \leq-\int_{X} f^{2}\langle\widetilde{W}, \theta\rangle d V_{g}+\int_{X}|\nabla f|^{2}|\widetilde{W}|^{2} d V_{g}
\end{aligned}
$$

By a simple density argument using Inequality (2.8), it can be shown that Estimate (2.10) still holds for any function $f$ such that $f,|\nabla f|=O\left(\mathrm{e}^{-b \rho}\right)$ for some $b>\frac{n-1}{2}-\frac{n-1}{p}$. We choose $f=f_{R}(\rho)$ where $f_{R}$ is a 1-parameter family of functions defined as follows:

$$
f_{R}(\rho):= \begin{cases}\mathrm{e}^{a \rho} & \text { if } \rho \leq R \\ \mathrm{e}^{a R-b(\rho-R)} & \text { if } \rho \geq R\end{cases}
$$

It is easy to see that these functions are Lipschitz continuous and satisfy $f,|\nabla f|=O\left(\mathrm{e}^{-b \rho}\right)$. From the fact that $|\nabla f|^{2}=a^{2} f^{2}$ if $\rho<R$ and $|\nabla f|^{2}=$ $b^{2} f^{2}$ if $\rho>R$, we get:

$$
\begin{aligned}
& {\left[\frac{(n-5)^{2}}{4}-a^{2}-C(n, \varepsilon)\right] \int_{\mathbb{D}_{R}} f^{2}|\widetilde{W}|^{2} d V_{g}} \\
& \quad+\left[\frac{(n-5)^{2}}{4}-b^{2}-C(n, \varepsilon)\right] \int_{X \backslash \mathbb{D}_{R}} f^{2}|\widetilde{W}|^{2} d V_{g} \\
& \quad \leq-\int_{X} f^{2}\langle\widetilde{W}, \theta\rangle d V_{g} .
\end{aligned}
$$


Choosing $b<\frac{n-5}{2}$, which is possible since $\frac{n-1}{2}-\frac{n-1}{p}<\frac{n-5}{2}$, and $\varepsilon$ so small that $\frac{(n-5)^{2}}{4}-b^{2}-C(n, \varepsilon) \geq 0$, we obtain

$$
\left[\frac{(n-5)^{2}}{4}-a^{2}-C(n, \varepsilon)\right] \int_{\mathbb{D}_{R}} \mathrm{e}^{2 a \rho}|\widetilde{W}|^{2} d V_{g} \leq-\int_{X} \mathrm{e}^{2 a \rho}\langle\widetilde{W}, \theta\rangle d V_{g} .
$$

Letting $R$ tend to infinity, and upon reducing the value of $\varepsilon$ so that $\frac{(n-5)^{2}}{4}-a^{2}-C(n, \varepsilon)>0$, we finally get

$$
\int_{\mathbb{D}_{R}} \mathrm{e}^{2 a \rho}|\widetilde{W}|^{2} d V_{g}<\infty
$$

This ends the proof of Proposition 2.13.

\section{Pointwise estimate for the Weyl tensor}

In this section, we assume that $\left(X^{n}, g\right)$ is an AHE manifold with Weyl tensor satisfying $\|\mathrm{W}\|_{L^{p}(X, g)}<\infty$ for some $p \leq \frac{n-1}{2}$. Note that on Einstein manifolds we always have $\mathrm{W}=\mathrm{Rm}-\mathbf{K}$. The main purpose of this section is to give a pointwise decay estimate for $\mathrm{W}$. We achieve this by two steps: first, we get the estimate by assuming $\left(X^{n}, g\right)$ is a $C^{2, \mu}$-conformally compact Einstein manifold. Obviously, even in this case the result has its own interests. Later, we remove the condition of $C^{2, \mu}$-regularity and try to obtain the pointwise estimate of $|\mathrm{W}|$ in more general situations. Unfortunately, due to some technical reasons mentioned in the introduction, we have to assume $n \geq 7$ in this case.

We begin by recaling the definition of a conformally compact manifold:

Definition 3.1. We say that $(X, g)$ is a $C^{k, \alpha}$-conformally compact manifold if

- there exists a smooth manifold $\bar{X}$ with boundary $\partial X$ whose interior is $X: X=\bar{X} \backslash \partial X$,

- and for some defining function $r, \bar{g}=r^{2} g$ extends to a $C^{k, \alpha}$ metric on $\bar{X}$

where a defining function $r$ is a smooth function $r: \bar{X} \rightarrow \mathbb{R}_{+}$such that $r^{-1}(0)=\partial X$ with $d r \neq 0$ at every point of $\partial X$.

Furthermore, assuming that $\sec _{g} \rightarrow-1$ at infinity, the function $r$ satisfies $|d r| \frac{2}{g} \equiv 1$ on $\partial X . C^{k, \alpha}$-conformally compact manifolds whose curvature tends 
to -1 at infinity are called asymptotically hyperbolic. We refer the reader to [22] and references therein for more details on these manifolds.

In order to get the pointwise decay of $\mathrm{W}$ which is mentioned above, we need the following lemma, which was observed in [28].

Lemma 3.2. Suppose that $\left(X^{n}, g\right)$ is a conformally compact Einstein manifold of regularity $C^{2}$. If its conformal infinity is conformally flat, then

$$
|\mathrm{W}|=O\left(r^{n+1}\right),
$$

where $r$ is the defining function determined by some conformal infinity.

Here is the outline of the proof of the above lemma. We refer the reader to [28] for details. Straightforward calculations yield that if an Einstein metric $g$ is at least $C^{2}$ conformally compact, then the sectional curvature in $X$ satisfies

$$
\sec _{g}=-1+O\left(r^{2}\right)
$$

The most basic and important fact about asymptotically hyperbolic manifolds is that a conformal infinity $\left(\partial X, g_{0}\right)$ determines a unique defining function $r$ in a collar neighborhood of $\partial X$ such that

$$
g=r^{-2}\left(d r^{2}+g_{r}\right)
$$

where $g_{r}$ is an $r$-dependent family of metrics on $\partial X$ with $\left.g_{r}\right|_{r=0}=g_{0}$. See, e.g., [21]. It follows from the work of Fefferman and Graham [13] that the Einstein equation implies the following asymptotic expansion for the metric $g$. For $n$ even,

$$
g_{r}=g_{0}+g_{(2)} r^{2}+(\text { even powers })+g_{(n-2)} r^{n-2}+g_{(n-1)} r^{n-1}+\cdots
$$

where the $g_{(j)}$ are tensors on $\partial X$ and $g_{(n-1)}$ is trace-free with respect to $g_{0}$. The tensors $g_{(j)}$ for $j \leq n-2$ are locally formally determined by the metric $g_{0}$, but $g_{(n-1)}$ is formally undetermined. For $n$ odd the analogous expansion is

$$
g_{r}=g_{0}+g_{(2)} r^{2}+(\text { even powers })+k r^{n-1} \log r+g_{(n-1)} r^{n-1}+\cdots
$$

where the $g_{(j)}$ 's are locally determined for $j \leq n-2, k$ is locally determined and trace-free, but $g_{(n-1)}$ is formally undetermined. 
Due to Theorem $\mathrm{A}$ in [11], we know that if $\left(X^{n}, g\right)$ is a conformally compact of regularity $C^{2}$ and its conformal infinity is smooth, then in fact $\left(X^{n}, g\right)$ is conformally compact of order $C^{\infty}$ if $n$ is even or if $n$ is odd and $k \equiv 0$, where $k$ is a conformally covariant tensor. Therefore according to the Fefferman-Graham expansion, we can get that $|\mathrm{W}|_{g}=O\left(r^{n+1}\right)$ if the conformal infinity is locally conformally flat.

Theorem 3.3. Suppose that $\left(X^{n}, g\right)$ is a conformally compact Einstein manifold of dimension $n \geq 5$ and of regularity $C^{2, \mu}$ for some $\mu \in(0,1)$. If we further suppose that there exists $p \in\left(1, \frac{n-1}{2}\right]$ such that $\|\mathrm{W}\|_{L^{p}\left(X^{n}, g\right)}<\infty$, then

$$
|\mathrm{W}|=O\left(r^{n+1}\right)
$$

where $r$ is some special defining function.

Proof. Let $r: \bar{X} \rightarrow \mathbb{R}$ be an arbitrary defining function for the conformal infinity $\partial X$ of $X$ and let $\bar{g}=\rho^{2} g$ be the compactified metric. We denote with a bar quantities associated to the metric $\bar{g}$, e.g., $\overline{\mathrm{W}}$ denotes its Weyl tensor. By assumption, $\bar{g}$ is a $C^{2, \mu}$ metric on $\bar{X}=X \cup \partial X$.

We first note that $|\mathrm{W}|_{g}=\rho^{2}|\overline{\mathrm{W}}|_{\bar{g}}$. As a consequence,

$$
\int_{X}|\mathrm{~W}|_{g}^{p} d V_{g}=\int_{X} \rho^{2 p-n}|\overline{\mathrm{W}}|_{\bar{g}}^{p} d V_{\bar{g}}
$$

Since $\bar{g}$ is $C^{2, \mu}, \overline{\mathrm{W}}$ is a continuous 4 -tensor. From the fact that $p \leq \frac{n-1}{2}$, the function of $\rho$ appearing in the integral on the right-hand side blows up faster than $\rho^{-1}$ when approaching $\partial X$. As a consequence, if this quantity is to be finite, this impose that $\overline{\mathrm{W}} \equiv 0$ on $\partial X$.

From the Fefferman-Graham expansion of the metric $g$, we immediately see that the second fundamental form of $\partial X$ in the manifold $\bar{X}$ vanishes.

If $\widehat{g}$ denotes the metric induced on the conformal infinity $\partial X$, it follows from the Gauss-Codazzi equations that the Riemann tensor $\widehat{\mathrm{Rm}}$ of $\widehat{g}$ is equal to the restriction of $\overline{\mathrm{Rm}}$ to $T(\partial X)$. We denote $\bar{P}$ and $\widehat{P}$ the Schouten tensors of the metrics $\bar{g}$ and $\widehat{g}$. From the decomposition of the Riemann tensors

$$
\left\{\begin{array}{l}
\widehat{\mathrm{Rm}}=\widehat{g} \oplus \widehat{P}+\widehat{\mathrm{W}}, \\
\overline{\mathrm{Rm}}=\bar{g} \otimes \bar{P}+\overline{\mathrm{W}}
\end{array}\right.
$$


it follows that

$$
\widehat{\mathrm{W}}=\overline{\mathrm{W}}+(\bar{P}-\widehat{P}) \otimes \widehat{g},
$$

Since $\overline{\mathrm{W}} \equiv 0$ on $\partial X$, we conclude that

$$
\widehat{\mathrm{W}}=(\bar{P}-\widehat{P}) \oplus \widehat{g}
$$

which implies $\widehat{W} \equiv 0$ because the two sides of the equality belong to orthogonal subspaces of $\widetilde{\Sigma}^{4}(\partial X)$.

As a consequence, we have proven that $\partial X$ is locally conformally flat. The theorem follows from Lemma 3.2.

Finally, we remove the condition $C^{2, \mu}$-regularity to give the pointwise estimate of $|\mathrm{W}|$. According to Proposition 2.13 we get weighted $L^{2}$-estimate for the Weyl tensor. Using Lemma 2.5, we are able to show the following theorem:

Theorem 3.4. Suppose that $\left(X^{n}, g\right), n \geq 7$ is a complete non-compact Einstein manifold with an essential set $\mathbb{D}$. If $\|\mathrm{W}\|_{L^{p}\left(X^{n}, g\right)}<\infty$ for some $p \in\left(1, \frac{n-1}{2}\right)$, then

$$
|\mathrm{W}| \leq C \mathrm{e}^{-(n+1) \rho}
$$

An essential element in the proof of this theorem is [20, Theorem 1.2] which we recall here for the sake of completeness:

Proposition 3.5. Suppose that $\left(X^{n}, g\right)$ is a complete Riemannian manifold with an essential set $\mathbb{D}$. If the Riemann tensor satisfies the following assumptions:

$$
\begin{aligned}
|\mathrm{Rm}-\mathbf{K}| & =O\left(\mathrm{e}^{-a \rho}\right) \\
|\nabla \mathrm{Rm}| & =O\left(\mathrm{e}^{-a \rho}\right)
\end{aligned}
$$

for some constant $a>2$, there is a smooth closed manifold $\partial X$ and a smooth structure on $\bar{X}=X \cup \partial X$, such that setting $r=\mathrm{e}^{-\rho}$ and extending it by zero on $\partial X, r$ is a defining function for $\partial X$ and the metric $\bar{g}=r^{2} g$ extends to $a$ $C^{2, \mu}$-metric on the manifold $\bar{X}$ for some $\mu \in(0,1)$. That is to say $(X, g)$ is $C^{2, \mu}$-conformally compact.

Proof of Theorem 3.4. We will assume that $n \geq 8$ and indicate the modifications for $n=7$. The first step is to obtain an exponential (pointwise) 
decay of $|\mathrm{W}|$ at infinity. To this end, we set $\mathrm{W}_{1}:=\mathrm{e}^{a \rho} \mathrm{W}$ for some $a>0$ to be chosen later. From Equation (1.2), $\mathrm{W}_{1}$ satisfies

$$
\begin{aligned}
\triangle \mathrm{W}_{1} & =\left(\triangle \mathrm{e}^{a \rho}\right) \mathrm{W}+2\left\langle\nabla \mathrm{e}^{a \rho}, \nabla \mathrm{W}\right\rangle+\mathrm{e}^{a \rho} \triangle \mathrm{W} \\
& =\left[a^{2}+(n-1) a+o(1)\right] \mathrm{W}_{1}+2 a \mathrm{e}^{a \rho} \nabla_{\nabla \rho} \mathrm{W}+\mathrm{e}^{a \rho} \triangle \mathrm{W} \\
& =\left[a^{2}+(n-1)(a-2)+o(1)\right] \mathrm{W}_{1}+2 a \mathrm{e}^{a \rho} \nabla_{\nabla \rho} \mathrm{W}-2 \mathcal{Q}\left(\mathrm{W}, \mathrm{W}_{1}\right),
\end{aligned}
$$

where we used $\triangle \rho=H=n-1+o(1)$ (see Lemma 2.4). Next, we compute $\triangle\left|\mathrm{W}_{1}\right|^{2}$ in two different ways at any point where $\left|\mathrm{W}_{1}\right| \neq 0$ :

$$
\begin{aligned}
\triangle\left|\mathrm{W}_{1}\right|^{2}= & 2\left(|\nabla| \mathrm{W}_{1}||^{2}+\left|\mathrm{W}_{1}\right| \triangle\left|\mathrm{W}_{1}\right|\right) \\
= & 2\left(\left|\nabla \mathrm{W}_{1}\right|^{2}+\left\langle\mathrm{W}_{1}, \triangle \mathrm{W}_{1}\right\rangle\right) \\
= & 2\left(\left|\nabla \mathrm{W}_{1}\right|^{2}+\left[a^{2}+(n-1)(a-2)+o(1)\right]\left|\mathrm{W}_{1}\right|^{2}\right. \\
& \left.+2 a \mathrm{e}^{a \rho}\left\langle\nabla_{\nabla \rho} \mathrm{W}, \mathrm{W}_{1}\right\rangle-2\left\langle\mathrm{~W}_{1}, \mathcal{Q}\left(\mathrm{W}, \mathrm{W}_{1}\right)\right\rangle\right) .
\end{aligned}
$$

As a consequence, we get the following equation for $\left|\mathrm{W}_{1}\right|$ :

$$
\begin{aligned}
& \left|\mathrm{W}_{1}\right| \triangle\left|\mathrm{W}_{1}\right|-\left[a^{2}+(n-1)(a-2)+o(1)\right]\left|\mathrm{W}_{1}\right|^{2} \\
& \quad=\left|\nabla \mathrm{W}_{1}\right|^{2}-\left.|\nabla| \mathrm{W}_{1}\right|^{2}+2 a \mathrm{e}^{2 a \rho}\left\langle\nabla_{\nabla \rho} \mathrm{W}, \mathrm{W}\right\rangle
\end{aligned}
$$

where we used Lemma 2.3 to get $\left\langle\mathrm{W}_{1}, \mathcal{Q}\left(\mathrm{W}, \mathrm{W}_{1}\right)\right\rangle=o\left(\left|\mathrm{~W}_{1}\right|^{2}\right)$.

The following refined Kato inequality holds for the Weyl tensor of any Einstein manifold (see, e.g., [9]):

$$
|\nabla| \mathrm{W}||^{2} \leq \frac{n-1}{n+1}|\nabla \mathrm{W}|^{2}
$$

We are going to take advantage of it to estimate the right-hand side of Equation (3.3). We first remark that

$$
\begin{aligned}
\left|\nabla \mathrm{W}_{1}\right|^{2} & =\mathrm{e}^{2 a \rho}|\nabla \mathrm{W}|^{2}+2 a \mathrm{e}^{2 a \rho}\left\langle\nabla_{\nabla \rho} \mathrm{W}, \mathrm{W}\right\rangle+a^{2} \mathrm{e}^{2 a \rho}|\mathrm{W}|^{2}, \\
\left.|\nabla| \mathrm{W}_{1}\right|^{2} & =\left.\mathrm{e}^{2 a \rho}|\nabla| \mathrm{W}\right|^{2}+2 a \mathrm{e}^{2 a \rho}\left\langle\nabla_{\nabla \rho}|\mathrm{W}|,|\mathrm{W}|\right\rangle+a^{2} \mathrm{e}^{2 a \rho}|\mathrm{W}|^{2} \\
& =\left.\mathrm{e}^{2 a \rho}|\nabla| \mathrm{W}\right|^{2}+a \mathrm{e}^{2 a \rho} \nabla_{\nabla \rho}|\mathrm{W}|^{2}+a^{2} \mathrm{e}^{2 a \rho}|\mathrm{W}|^{2} \\
& =\left.\mathrm{e}^{2 a \rho}|\nabla| \mathrm{W}\right|^{2}+2 a \mathrm{e}^{2 a \rho}\left\langle\nabla_{\nabla \rho} \mathrm{W}, \mathrm{W}\right\rangle+a^{2} \mathrm{e}^{2 a \rho}|\mathrm{W}|^{2} .
\end{aligned}
$$

Therefore, using Inequality (3.4), we get:

$$
\left|\nabla \mathrm{W}_{1}\right|^{2}-|\nabla| \mathrm{W}_{1}||^{2} \geq \frac{2}{n-1} \mathrm{e}^{2 a \rho}|\nabla| \mathrm{W}||^{2}
$$


Next, using Young's inequality, we remark that

$$
2 a \mathrm{e}^{2 a \rho}\left\langle\nabla_{\nabla \rho} \mathrm{W}, \mathrm{W}\right\rangle \geq-\left.\frac{2}{n-1} \mathrm{e}^{2 a \rho}|\nabla| \mathrm{W}\right|^{2}-\frac{n-1}{2} a^{2} \mathrm{e}^{2 a \rho}|\mathrm{W}|^{2} .
$$

Thus, Equation (3.3) yields the following differential inequality:

$$
\left|\mathrm{W}_{1}\right| \triangle\left|\mathrm{W}_{1}\right|-\left[a^{2}+(n-1)(a-2)+o(1)\right]\left|\mathrm{W}_{1}\right|^{2} \geq-\frac{n-1}{2} a^{2}\left|\mathrm{~W}_{1}\right|^{2}
$$

We select $a=\frac{n-1}{n-3}$. The previous inequality becomes

$$
\triangle\left|\mathrm{W}_{1}\right| \geq\left[-\frac{1}{2} \frac{(n-1)(3 n-11)}{n-3}+o(1)\right]\left|\mathrm{W}_{1}\right|
$$

at any point where $\left|\mathrm{W}_{1}\right|>0$. Note that when $n>7, a<\frac{n-5}{2}$ so from Proposition 2.13, $W_{1} \in L^{2}$. We claim that $\left|W_{1}\right| \leq C \mathrm{e}^{-\frac{n-1}{2} \rho}$. Indeed, set $b:=$ $\frac{n-1}{2}+\delta$ for some small $\delta>0$. Then we have

$$
\left(\triangle+\frac{1}{2} \frac{(n-1)(3 n-11)}{n-3}\right) \mathrm{e}^{-b \rho}=\left(\delta^{2}-\frac{1}{4} \frac{(n-1)(n-5)^{2}}{n-3}+o(1)\right) \mathrm{e}^{-b \rho} .
$$

Select $\varepsilon>0$ such that

$$
\varepsilon<\frac{1}{4} \frac{(n-1)(n-5)^{2}}{n-3} .
$$

Provided that $\delta^{2}<\frac{\varepsilon}{2}$, there exists a compact set $K \supset \mathbb{D}$ such that

$$
\left\{\begin{array}{l}
\triangle\left|\mathrm{W}_{1}\right| \geq-\left[\frac{1}{2} \frac{(n-1)(3 n-11)}{n-3}+\frac{\varepsilon}{2}\right]\left|\mathrm{W}_{1}\right| \\
\triangle \mathrm{e}^{-b \rho} \leq-\left[\frac{1}{2} \frac{(n-1)(3 n-11)}{n-3}+\frac{\varepsilon}{2}\right] \mathrm{e}^{-b \rho}
\end{array}\right.
$$

and such that for any $W^{1,2}$-function $\varphi$ supported in $K$, the following $L^{2}$-estimate holds (Lemma 2.5):

$$
\int_{X^{n}}|\nabla \varphi|^{2} d V_{g} \geq\left[\frac{(n-1)^{2}}{4}-\frac{\varepsilon}{2}\right] \int \varphi^{2} d V_{g}
$$

We set $\psi=\left|\mathrm{W}_{1}\right|-C \mathrm{e}^{-b \rho}$ where $C$ is chosen so large that $\psi<0$ on $K$. Then $\psi$ satisfies

$$
\triangle \psi \geq-\left[\frac{1}{2} \frac{(n-1)(3 n-11)}{n-3}+\frac{\varepsilon}{2}\right] \psi
$$


We also define $\psi_{+}=\max \{\psi, 0\}$ and note that $\psi_{+} \in W^{1,2}$ and $\operatorname{supp} \psi_{+} \subset$ $X \backslash K$. From Inequality (3.8), we get

$$
\begin{aligned}
{\left[\frac{(n-1)^{2}}{4}-\frac{\varepsilon}{2}\right] \int_{X}\left|\psi_{+}\right|^{2} d V_{g} } & \leq \int_{X^{n}}\left|\nabla \psi_{+}\right|^{2} d V_{g} \\
& \leq-\int_{X^{n}} \psi_{+} \Delta \psi d V_{g} \\
& \leq\left[\frac{1}{2} \frac{(n-1)(3 n-11)}{n-3}+\frac{\varepsilon}{2}\right] \\
& \times \int_{X^{n}}\left(\psi_{+}\right)^{2} d V_{g}, \\
{\left[\frac{1}{4} \frac{(n-1)(n-5)^{2}}{n-3}-\varepsilon\right] \int_{X^{n}}\left(\psi_{+}\right)^{2} d V_{g} } & \leq 0 .
\end{aligned}
$$

From our assumption on $\varepsilon$, this immediately implies that $\psi_{+} \equiv 0$, that is to say

$$
\left|\mathrm{W}_{1}\right| \leq C \mathrm{e}^{-b \rho}
$$

or equivalently,

$$
|\mathrm{W}| \leq C \mathrm{e}^{-\left(\frac{1}{2} \frac{(n-1)^{2}}{n-3}+\delta\right) \rho}
$$

Since $n \geq 8, \frac{1}{2} \frac{(n-1)^{2}}{n-3}>2$. In particular, from Proposition 3.5, we conclude that the manifold $\left(X^{n}, g\right)$ is $C^{2, \mu}$-conformally compact for some $\mu \in(0,1)$. So it falls into the assumptions of Theorem 3.3. This concludes the proof of Theorem 3.4 for $n \geq 8$.

If $n=7$, then $\frac{n-1}{n-3}=\frac{3}{2}>1=\frac{n-5}{2}$ so we can no longer apply Proposition 2.13 for this value of $a$. Instead we choose $a=\frac{n-5}{2}-\frac{1}{4}$. Inequality (3.6) becomes

$$
\triangle\left|\mathrm{W}_{1}\right| \geq\left[8+\frac{5}{8}+o(1)\right]\left|\mathrm{W}_{1}\right| .
$$

Setting $b:=3+\delta$, it can be checked that $\mathrm{e}^{-b \rho}$ satisfies

$$
\triangle \mathrm{e}^{-b \rho} \leq\left[8+\frac{5}{8}+o(1)\right] \mathrm{e}^{-b \rho},
$$

outside some compact subset. We can then rephrase the previous proof, the only point to note is that

$$
\frac{(n-1)^{2}}{4}=9>8+\frac{5}{8} .
$$

This is what allows the use of the asymptotic $L^{2}$-estimate (Lemma 2.5). 


\section{Applications}

Together with Theorem 1.2, the rigidity result [20, Theorem 1.6] implies

Theorem 4.1. Suppose that $\left(X^{n}, g\right), n \geq 7$ is a complete non-compact Einstein manifold with an essential set $\mathbb{D}$ and that $X^{n}$ is simply connected at infinity. If we further assume $\|\mathrm{W}\|_{L^{p}\left(X^{n}, g\right)}<\infty$ for some $p$ satisfying $1<p<\frac{n-1}{2}$, then $\left(X^{n}, g\right)$ is isometric to $\mathbb{H}^{n}$.

Proof. By Theorem 3.4, we know that there exists a constant $C>0$ such that

$$
|\mathrm{W}| \leq C \mathrm{e}^{-(n+1) \rho} .
$$

On the other hand, by a direct refinenemt of the proof of Lemma 2.3, we also have

$$
|\nabla \mathrm{W}| \leq C^{\prime} \mathrm{e}^{-(n+1) \rho}
$$

for some constant $C^{\prime}>0$. See also [3, Theorem 4.3]. The theorem then follows from the rigidity theorem [20, Theorem 5.1].

As another application, we consider a similar question for static vacuum spacetimes. We recall that an $(n+1)$-dimensional static spacetime $\left(N^{n+1}, g\right)$ is a solution of the vacuum Einstein equations

$$
\text { Ric }-\frac{\text { Scal }}{2} g+\Lambda g=0
$$

of the form

$$
\begin{aligned}
N^{n+1} & =\mathbb{R} \times M^{n}, \\
g & =-V^{2} d t^{2}+h
\end{aligned}
$$

where $\left(M^{n}, h\right)$ is a Riemannian manifold, $V$ is a positive function on $M^{n}$ and $\Lambda$ is the so-called cosmological constant which we choose equal to $-\frac{n(n-1)}{2}$. The vacuum Einstein equations can be written in terms of $h$ and $V$ as

$$
\operatorname{Ric}_{h}+n h=\frac{\operatorname{Hess}(V)}{V},
$$

and

$$
\triangle_{h} V=n V
$$

Computing the trace of these two equations, we see that $h$ has constant scalar curvature Scal $=-n(n-1)$. We will often just call the triple 
$\left(M^{n}, h, V\right)$ a static vacuum. We set

$$
\begin{aligned}
& \mathrm{E}:=\operatorname{Rm}_{h}-\mathbf{K}, \\
& \mathrm{T}:=\operatorname{Ric}_{h}+(n-1) h=\frac{\operatorname{Hess}(V)}{V}-h .
\end{aligned}
$$

As another application of Theorem 1.2, we state the following theorem:

Theorem 4.2. Suppose given $\left(M^{n}, h, V\right)$ a static vacuum with $n \geq 6$ such that $(M, h)$ has an essential set $\mathbb{D}$ and $\int_{M^{n}} V|E|_{h}^{p} d V_{h}<\infty$, for some $p \in$ $\left(1, \frac{n}{2}\right)$. If we further assume that $V$ is proper, then there exists a constant $C>0$ such that

$$
|E| \leq C \mathrm{e}^{-(n+2) \rho}
$$

and

$$
\left|\frac{\operatorname{Hess}(V)}{V}-h\right| \leq C \mathrm{e}^{-(n+2) \rho},
$$

where $\rho$ is the distance to the essential set $\mathbb{D}$.

Notice that for static vacuum $\left(M^{n}, h, V\right)$, the Riemannian metric $g=$ $V^{2} d \theta^{2}+h$ is an Einstein metric on $\mathbb{S}^{1} \times M$. Hence, we consider the Einstein manifold $\left(\mathbb{S}^{1} \times M, V^{2} d \theta^{2}+h\right)$. For convenience, in the following, the index 0 refers to the direction $\partial_{\theta}$. Latin indices take values 1 to $n$ and refer to coordinates on $M$.

In order to prove Theorem 4.2, we need the following two lemmas:

Lemma 4.3. Let $\left(M^{n}, h, V\right)$ be a static vacuum, if $\int_{M} V|E|_{h}^{p} d V_{h}<\infty$, then

$$
\int_{\mathbb{S}^{1} \times M}\left|\mathrm{~W}_{g}\right|_{g}^{p} d V_{g}<\infty .
$$

Proof. By a direct computation, using Equations (4.1) and (4.2), we get

$$
\begin{aligned}
& W_{i j k l}(g)=E_{i j k l}, \\
& W_{0 j k l}(g)=0, \\
& W_{0 j 0 l}(g)=-V^{2}\left(V^{-1} \nabla_{h}^{2} V-h\right)=-V^{2} T,
\end{aligned}
$$

and

$$
|\mathrm{W}(g)|_{g}^{2}=|E|_{h}^{2}+4|T|_{h}^{2} .
$$

Note that $T_{i k}=h^{j l} E_{i j k l}$, thus there is a constant $C=C(n)$ such that

$$
|E|_{h} \leq\left|\mathrm{W}_{g}\right|_{g} \leq C|E|_{h}
$$


Therefore the assumption

$$
\int_{M^{n}} V|E|_{h}^{p} d V_{h}<\infty
$$

is equivalent to

$$
\int_{S^{1} \times M^{n}}\left|\mathrm{~W}_{g}\right|_{g}^{p} d V_{g}<\infty
$$

Lemma 4.4. Let $\left(M^{n}, h, V\right)$ be a static vacuum. If $\left(M^{n}, h\right)$ has an essential set $\mathbb{D}$ and if $V$ is a proper function, the manifold $\left(\mathbb{S}^{1} \times M^{n}, g\right)$ admits an essential set.

Proof. We first remark that since $h$ has constant scalar curvature and negative sectional curvature outside $\mathbb{D}$, it has bounded sectional curvature. From Lemma 2.2, we know that $\left(M^{n}, h\right)$ has bounded sectional curvature. It follows from the work of Anderson [1] (see also [19]) that there exists an $r_{0}>0$ such that for any given point $x \in M$ there exists an harmonic coordinate system $\left(y^{i}\right)$ on $B_{r_{0}}(x)$ such that

$$
\left\{\begin{array}{l}
\frac{1}{2} \delta_{i j} \leq h_{i j} \leq 2 \delta_{i j} \\
\sup _{y}\left|\partial_{k} h_{i j}(y)\right|+\sup _{y \neq z} \frac{\left|\partial_{k} h_{i j}(y)-\partial_{k} h_{i j}(z)\right|}{|y-z|^{\frac{1}{2}}} \leq 1
\end{array}\right.
$$

where $h_{i j}$ denotes the components of the metric $h$ in the coordinate system $\left(y^{i}\right)$. It follows from Equation (4.2) and standard elliptic regularity in the coordinate system $\left(y^{i}\right)$ that there exists a constant $C$ such that for any point $x \in M$,

$$
\|d V\|_{L^{\infty}\left(B_{r_{0} / 3}(x)\right)}+\|\operatorname{Hess} V\|_{L^{\infty}\left(B_{r_{0} / 3}(x)\right)} \leq C\|V\|_{L^{\infty}\left(B_{r_{0} / 2}(x)\right)} .
$$

From Harnack estimate (see, e.g., [17, Corollary 9.25]), we deduce that there exists a constant $C^{\prime}$ such that

$$
\|V\|_{L^{\infty}\left(B_{r_{0} / 3}(x)\right)}+\|d V\|_{L^{\infty}\left(B_{r_{0} / 3}(x)\right)}+\|\operatorname{Hess} V\|_{L^{\infty}\left(B_{r_{0} / 3}(x)\right)} \leq C^{\prime} \inf _{\left.B_{r_{0} / 3}(x)\right)} V .
$$

We set $H:=V T=$ Hess $V-V h$ and remark that $H$ is traceless and satisfies

$$
\nabla_{i} H_{j k}-\nabla_{j} H_{i k}=-\mathrm{E}_{k i j}^{l} \nabla_{l} V
$$


We select an arbitrary $q>n$. From [16, Proposition 2.6] and the Sobolev embedding theorem, there exists a constant $C^{\prime}$ such that

$$
\|H\|_{L^{\infty}\left(B_{r_{0} / 4}(x)\right)} \leq C^{\prime \prime}\left(\|H\|_{L^{q}\left(B_{r_{0} / 3}(x)\right)}+\|E * \nabla V\|_{L^{q}\left(B_{r_{0} / 3}(x)\right)}\right),
$$

where $E * \nabla V$ denotes the tensor appearing on the right-hand side of Equation (4.4). It then follows from Estimate (4.3) that for some constant $C^{(3)}$,

$$
\begin{aligned}
\|T\|_{L^{\infty}\left(B_{r_{0} / 4}(x)\right)} & \leq C^{(3)}\left(\|T\|_{L^{q}\left(B_{r_{0} / 3}(x)\right)}+\|E\|_{L^{q}\left(B_{r_{0} / 3}(x)\right)}\right) \\
& \leq C^{(3)}\left(\|\operatorname{Ric}+(n-1) h\|_{L^{q}\left(B_{r_{0} / 3}(x)\right)}+\|E\|_{L^{q}\left(B_{r_{0} / 3}(x)\right)}\right) .
\end{aligned}
$$

Since $V$ is a proper positive function, it is bounded from below by 1 outside a compact set. So $E$ and Ric $+(n-1) h$ (which is the trace of $E$ ) belong to $L^{p}$. It is then straightforward to adapt the argument of Lemma 2.3 to conclude from the previous estimate that $|T|$ tends uniformly to zero at infinity. In particular, $\operatorname{Hess}^{h} V$ is positive definite outside some compact set. Hence, $\operatorname{Hess}^{g} V$ being given by

$$
\begin{aligned}
\operatorname{Hess}_{i j}^{g} V & =\operatorname{Hess}_{i j}^{h} V, \\
\operatorname{Hess}_{i 0}^{g} V & =0, \\
\operatorname{Hess}_{00}^{g} V & =V|d V|_{h}^{2},
\end{aligned}
$$

$V$ also has non-negative Hessian with respect to the metric $g$ outside some compact set.

As another consequence of our estimate, the sectional curvature of $g$ tends to -1 at infinity. It follows from [14, Lemma 2.5.11] that $\left(\mathbb{S}^{1} \times M^{n}, g\right)$ admits an essential set.

Theorem 4.2 is then a consequence of Theorem 3.4 applied to the metric $g=V^{2} d \theta^{2}+h$.

If we further assume that $M$ is spin, then we fall into the assumptions of [29, Theorem 1] (See also Theorem 1.2 in [26]) so we get the following theorem:

Theorem 4.5. Suppose that $\left(M^{n}, h, V\right)$ is a static vacuum with $n \geq 6$. Assume further that

1. $M$ is spin,

2. $(M, h)$ has an essential set $\mathbb{D}$, 
3. $V$ is a proper function,

4. and $\int_{M^{n}} V|E|_{h}^{p} d V_{h}<\infty$ for some $p \in\left(1, \frac{n}{2}\right)$

then $\left(M^{n}, h\right)$ is the hyperbolic space and $V=\cosh (r)$, where $r$ is the distance function to a certain point $x_{0} \in M$. Equivalently, the spacetime $(\mathbb{R} \times$ $\left.M,-V^{2} d t^{2}+h\right)$ is the anti-deSitter space.

\section{Acknowledgments}

The authors are grateful to Professor Jie Qing, Dr. Jie Wu and Dr. Xue $\mathrm{Hu}$ for their interest in this work and for many enlightening discussions. The authors would also like to warmly thank both referees for numerous suggestions which helped to improve the presentation. This work was partially supported by the NSF grant of China 10990013.

\section{References}

[1] M.T. Anderson, Convergence and rigidity of manifolds under Ricci curvature bounds, Invent. Math. 102(2) (1990), 429-445.

[2] E. Bahuaud, Intrinsic characterization for Lipschitz asymptotically hyperbolic metrics, Pacific J. Math. 239(2) (2009), 231-249.

[3] E. Bahuaud and R. Gicquaud, Conformal compactification of asymptotically locally hyperbolic metrics, J. Geom. Anal. 21(4) (2011), 1085-1118.

[4] E. Bahuaud and T. Marsh, Hölder compactification for some manifolds with pinched negative curvature near infinity, Can. J. Math. 60(6) (2008), 1201-1218.

[5] S. Bando, A. Kasue and H. Nakajima, On a construction of coordinates at infinity on manifolds with fast curvature decay and maximal volume growth, Invent. Math. 97(2) (1989), 313-349.

[6] A.L. Besse, Einstein manifolds. In Classics in Mathematics, SpringerVerlag, Berlin, 2008, Reprint of the 1987 edition.

[7] O. Biquard, Asymptotically symmetric Einstein metrics, SMF/AMS Texts and Monographs, vol. 13, American Mathematical Society, Providence, RI, 2006, Translated from the 2000 French original by Stephen S. Wilson. 
[8] J.-P. Bourguignon, Les variétés de dimension 4 à signature non nulle dont la courbure est harmonique sont d'Einstein, Invent. Math. 63(2) (1981), 263-286.

[9] D.M.J. Calderbank, P. Gauduchon and M. Herzlich, Refined Kato inequalities and conformal weights in Riemannian geometry, J. Funct. Anal. 173(1) (2000), 214-255.

[10] J. Cheeger and G. Tian, On the cone structure at infinity of Ricci flat manifolds with Euclidean volume growth and quadratic curvature decay, Invent. Math. 118(3) (1994), 493-571.

[11] P.T. Chruściel, E. Delay, J.M. Lee and D.N. Skinner, Boundary regularity of conformally compact Einstein metrics, J. Differ. Geom. 69(1) (2005), 111-136.

[12] J.F. Escobar and A. Freire, The differential form spectrum of manifolds of positive curvature, Duke Math. J. 69(1) (1993), 1-41.

[13] C. Fefferman and C. Robin Graham, Conformal invariants, Astérisque (1985), no. Numero Hors Serie, 95-116, The Mathematical Heritage of Élie Cartan (Lyon, 1984).

[14] R. Gicquaud, Etude de quelques problèmes d'analyse et de géométrie sur les variétés asymptotiquement hyperboliques, $\mathrm{PhD}$ thesis, Université Montpellier 2, 2009.

[15] _ Linearization stability of the Einstein constraint equations on an asymptotically hyperbolic manifold, J. Math. Phys. 51 (2010), 14.

[16] Conformal compactification of asymptotically locally hyperbolic metrics II: Weakly ALH metrics, http://arxiv.org/abs/1109.5096, 2011.

[17] D. Gilbarg and N.S. Trudinger, Elliptic partial differential equations of second order, Classics in Mathematics, Springer-Verlag, Berlin, 2001, Reprint of the 1998 edition.

[18] C. Robin Graham and J.M. Lee, Einstein metrics with prescribed conformal infinity on the ball, Adv. Math. 87(2) (1991), 186-225.

[19] E. Hebey and M. Herzlich, Harmonic coordinates, harmonic radius and convergence of Riemannian manifolds, Rend. Mat. Appl. (7) 17(4) (1997), 569-605 (1998).

[20] X. Hu, J. Qing and Y. Shi, Regularity and rigidity of asymptotically hyperbolic manifolds, Adv. Math. 230 (2012), 2332-2363. 
[21] J.M. Lee, The spectrum of an asymptotically hyperbolic Einstein manifold, Commun. Anal. Geom. 3(1-2) (1995), 253-271.

[22] _ Fredholm operators and Einstein metrics on conformally compact manifolds, Mem. Amer. Math. Soc. 183(864) (2006), vi+83.

[23] H. Li and H. Yin, On stability of the hyperbolic space form under the normalized Ricci flow, Int. Math. Res. Not. IMRN (2010), 15(15), 2903-2924.

[24] P. Li and J. Wang, Weighted Poincaré inequality and rigidity of complete manifolds, Ann. Sci. École Norm. Sup. (4) 39(6) (2006), 921-982.

[25] P. Petersen, Riemannian geometry, 2nd ed., Graduate Texts in Mathematics, vol. 171, Springer, New York, 2006.

[26] J. Qing, On the uniqueness of AdS space-time in higher dimensions, Ann. Henri Poincaré 5(2) (2004), 245-260.

[27] Y. Shi and G. Tian, Rigidity of asymptotically hyperbolic manifolds, Commun. Math. Phys. 259(3) (2005), 545-559.

[28] X. Wang, On conformally compact Einstein manifolds, Math. Res. Lett. 8(5-6) (2001), 671-688.

[29] On the uniqueness of AdS spacetime in any dimension, Acta Math. Sin. (Engl. Ser.), 21, 2005, pp. 917-922.

Laboratoire de Mathématiques et de Physique Théorique

UFR SCIENCES ET TECHNOLOGIE

Université François Rabelais Parc de Grandmont 37300 Tours

FRANCE

E-mail address: romain.gicquaud@lmpt.univ-tours.fr

Key Laboratory of Pure and Applied mathematics

School of Mathematics Science

PEKING UNIVERSITY

BeIJING 100871

PeOple's Republic of ChinA

E-mail address: jidandan@pku.edu.cn; ygshi@math.pku.edu.cn

ReCEIVED OCTOBer 28, 2012 
